\title{
Application of mobile cloud computing in emergency health care
}

\author{
Shihab A. Hameed, Ali Nirabi, Mohamed Hadi Habaebi, Alaa Haddad \\ Department of Electrical and Computer Engineering, International Islamic University Malaysia, Malaysia
}

\begin{tabular}{l}
\hline Article Info \\
\hline Article history: \\
Received Oct 2, 2018 \\
Revised Jan 10, 2019 \\
Accepted Feb 1, 2019 \\
\hline
\end{tabular}

\section{Keywords:}

Cloud computing

Cross-platform

Emergency

Health care

Mobile cloud computing for emergency healthcare model (MCCEH)

\begin{abstract}
Mobile applications in emergency health care help maintain patient confidentiality and manage patient records, data storage. Compiles and analyzes care of better quality care. new implementations come with new goals and technologies like using mobile application with cloud computing system and reducing the responding time to safe the patient life and give the patient best health care professional service transition to using of mobile application in emergency healthcare, this paper will present (MCCEH) mobile cloud computing in emergency health care model, mainly reducing the wasting time in emergency health care, The process starting once the accident occurred and the patient run the application, mobile application will detect the patient location and allow him to book nearest medical center or specialist in some emergency cases once the patient did the booking will send help request to medical center this process will include an online preregister patient in the medical center to save time of patient registration, MCCEH model allows the patients to review the previous feedback and experiences of each specialist or medical center and allows doctors to be able to stay in contact with their patients more often and by communication through mobiles applications and share messages and photos of the accident or emergency case itself.
\end{abstract}

Copyright $(2) 2019$ Institute of Advanced Engineering and Science. All rights reserved.

\section{Corresponding Author:}

Ali Nirabi,

Department of Electrical and Computer Engineering,

International Islamic University Malaysia,

Jalan Gonmak, 53100, Kuala Lumpur, Malaysia.

Email: nirabi.ali@live.iium.edu.my

\section{INTRODUCTION}

Health Information System can help to increase the patient safety alerts creating less room for error and better documentation for doctors and nurses, the challenges in health care system is to makesure are the healthcare services are always running keep the health care application up to date, the exsiting health care systems are not scalable enough for the increasing number of patients or to receive the request from patient instantly almost health care systems those using the dedicated or physical server it possible to be down any time [1]. Cloud computing promises low cost, high scalability, instantly access for data records [2, 3], availability and disaster recover-ability which can be a natural solution for some of the problems faced in storing and analyzing patients' medical records [4].

Usually in the mobile developments, the developers using a specific programming language and specific platform for a target mobile operating system and then will do the same if the the requirements request new application for different mobile system operating systems to ensure the less cost and saving time for health care mobile development will implement cross platform development, Cross platform development refers to a single code can able to deploy it on different operating systems. E.g. IOS, Android, windows ...etc, it could be develop one code in one platform and able to deploy it for multiple mobile operating systems [5]. 
The goal of a paper is to design MCCEH model using cloud computing server and store all records and health care emergency services in cloud server, allowing the patient find nearest medical center and do preregister in the hospital in instant time and also allow the patient to get help from nearest specialist and set appointment.

\section{RELATED WORKS}

In the paper presented a health care novel system [6] that allows mobile to users to have access to healthcare services in the case of emergencies the limitation of this paper. There is no notification system implemented yet while it will help the specialist to get any request or message in real time while in MCCEH model will implement the notification system to notify the user with any update or message in inbox [7]. This study has followed same previous existing emergency system with focusing to reduce Time of arrival emergency to accident location the limitation of this study In some cases the system won't be useful for the user when the users haven't enough mobile balance to have a call or send SMS message to make emergency request [8], while in $\mathrm{MCCEH}$ model will implement chat system between the users using cloud computing system [9]. This paper have proposed the E-Ambulance framework, which is a smart ambulance system model that provides health monitoring of patients for remote medical professionals, the limitation of this work is the security, The system proposed the data will be store in database and hosted in dedicated server the security will be issue for this important data it can be hacked or misused as the dedicate server is physically accessible, instead of being hosted on physical hardware the MCCEH model will override this issue by using cloud computing server "virtualized" environment that's managed by your cloud hosting provider. The paper [9] presented a PHR-based EMS in a cloud computing platform focusing on an Android application, the limitation of this work, In the proposed system architecture proposed the development directed for android so the application will be linked to one singular environment only, while in these days there are a lot of various mobile operating systems users this paper will override this weakness by using cross platform (build one application and deploy in different several mobile operating systems) proposed solution MCCEH model and design goals.

\section{RESEARCH METHOD}

There are many studied related to emergency health care and cloud computing have been published but in the fact there are very limited number of successful articles and implementation not yet exist for the most, In this study will design mobile cloud computing in emergency health care MCCEH the main objective is to reduce Time of arrival emergency to accident's location the limitation, using the through global positioning system (GPS) will implement Google services to help the user to get the nearest medical center / specialists which able to communicate the specialist and get initial diagnose and set appointment if need it, the model will focus on creating the friendly mobile application which can be efficient in emergency cases, clicking single action the information will send to medical center instantly, Instead of traditional emergency way that following to force the user in the accident to decide which department have to be connected and then have to find out their contact numbers which may it be wasting the victim life while.

Our research defines MCCHE model containing three main components 1- cloud computing system containing all the hosted the emergency services and the database, 2- two different types of users 3- mobile application which allow the user to user the emergency services, The MCCEH architecture presented the interaction between the three levels of users Administrator, Specialist and patient/normal user, once the user run the application the application will detecting his current location and then the user will able to find nearest medical center and send help request, emergency services in the cloud computing server will send the request with the accident's location and patient information in instant time.

\subsection{MCCEH system flow chart}

Figure 1 is showing flow shart of MCCEH model flow chart, The process will start when the user run the application will redirect to login interface can choose user type is;

a. Patient user : the system will detect the current location and then will able to search to nearest specialist or medical center, Then will get list of result and may able to contact and make appointment with specialist.

b. Specialist users: will be asked to have credential to login to system and will responsible to update profile, receive messages and request for appointments.

c. System administrator: this user is responsible to manage whole data in the system;

1) add/remove/approve specialists

2) Add/remove medical centers

3) Manage database data 


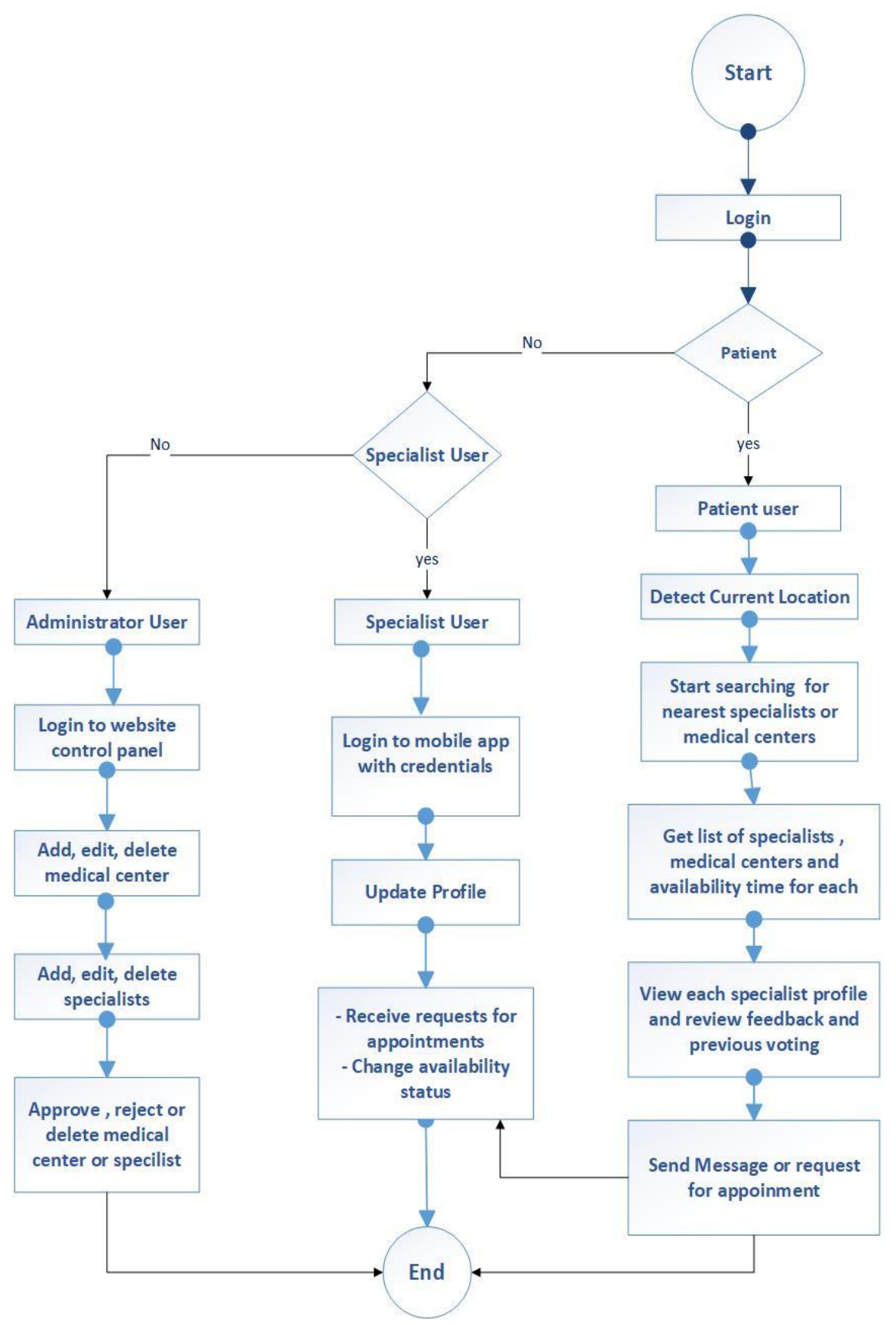

Figure 1. MCCEH flow chart

\subsection{Model architecture}

The MCCEH model consists of three main components as showed in Figure 2:

a. Database to store all the data medical centers, specialists and clinics

b. Web services to manage all data in the model and handle the requests from the user either is the specialist/ medical center or patient user like request for approve new specialists and verified the profile and accept it or decline and the help request sended from the patient user and get the appointment for initial diagnoses, host the other services in cloud computing system to get and send data from database.

c. Mobile application the patient user, clinic users medical centers can use it, the mobile application will integrate with web services to get and post data to database 




Figure 2. MCCEH Model architecture

\subsubsection{MCCEH cloud computing}

The term of cloud computing is refer to computing resources available on demand to a shared pool of configurable computing resources such as servers, storage, and applications [10]. Cloud computing promises low cost, high scalability, instantly access for data records, availability and disaster recover-ability which can be a natural solution for some of the problems faced in storing and analyzing patients' medical records.

This paper examines the impact of cloud computing on improving health care services. More specifically to help the patients in accidents location and before arrive to hospital.

Cloud computing has the great ability to offer infinite capacity and power of process in the emergency health care sector [11-13]. This leads computer to be used efficiently and exclusively by the sharing of resources in health care. In MCCEH using the cloud computing to host all medical center, specialists and users information records, all emergency service will be hosted on the cloud and then will able to get the services on demand using mobile application.

\subsubsection{MCCEH users}

The system architecture is shown in Figure 1, consists of two different types of users:

a. Specialist User able login to a system or register create new account using the mobile application, and will get the approval or rejection from an system administrator, after getting the approval will be able to:

1) Complete the profile with in personal information, location address information, availability schedule times.

2) In the case the specialist is available will able to receive notification appointments booking request or messages.

b. Normal user (patient) :

1) In the accident case the patient will able to find the nearest medical centre and book ambulance

2) If a member of the family has a health problem in late time like dental, fever or some pain but the user will be able to find nearest available specialist from his location and can register him self in the medical centers/specialist and set the appointment.

3) The user able to search by his current location and specialist category like Family Dental, Physicians, Neurologists, Gastroenterologists, etc.

\subsubsection{MCCEH mobile application}

The third part of MCCEH Architecture component is Mobile Application. Designing friendly Mobile Cloud computing emergency health care application will allow to users searching for nearest specialists or medical center and allow to send request for emergency or set appointments the process will start will initial interface to choose whether is patient user or specialist user as shown in Figure 3. If the user is patient/ normal user once click login button the application will detect the current location for the user as it will progress the process once the help request has sent as shown in Figure 4.

The next step is asking the user whether he is looking for emergency in medical center or setting appointment with specialist then will allow the user to choose the specialization to proceed the searching process with one minute and less the user will have the result on his mobile screen as showing in Figure 5. The user able to contact the specialist. 


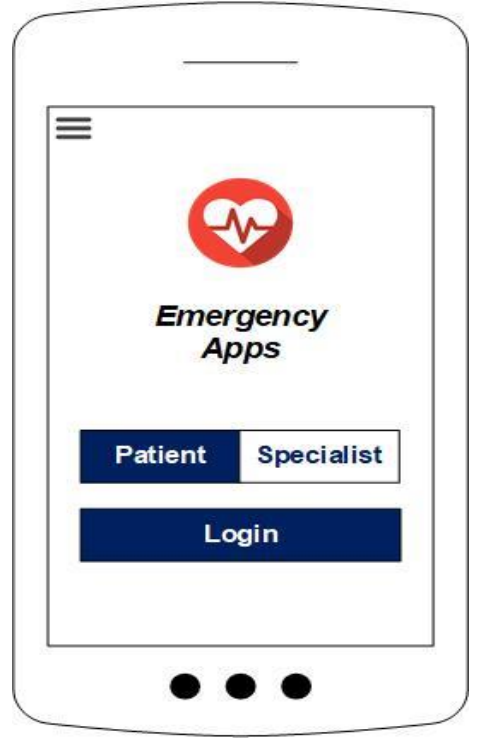

Figure 3. Initial interface

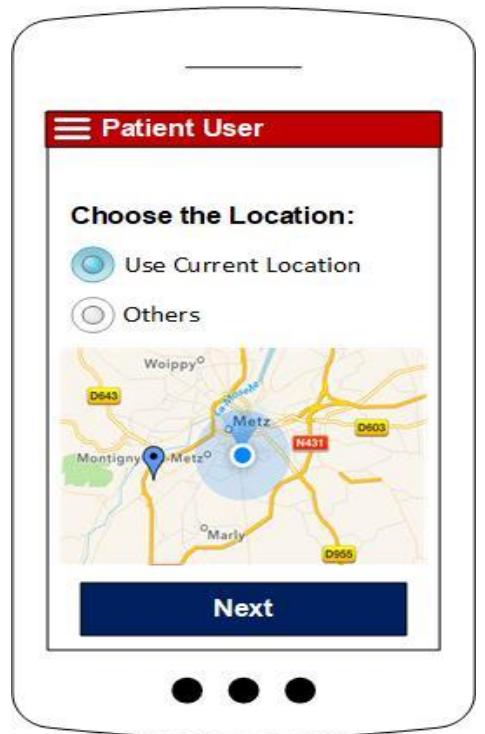

Figure 4. Detecting current location



Figure 5. Search process

The user able to contact the specialist and ask for initial diagnose or set appointment if need. In the result interface will show listing containing the availability time and previous feed back have been comments about each specialists. In case the user is specialist then will direct him to login/registration interface as show in Figure 6. After the registration been done and the specialist will able to login his account and update his profile, check messages, and receive request for appointments as show in Figure 7. 


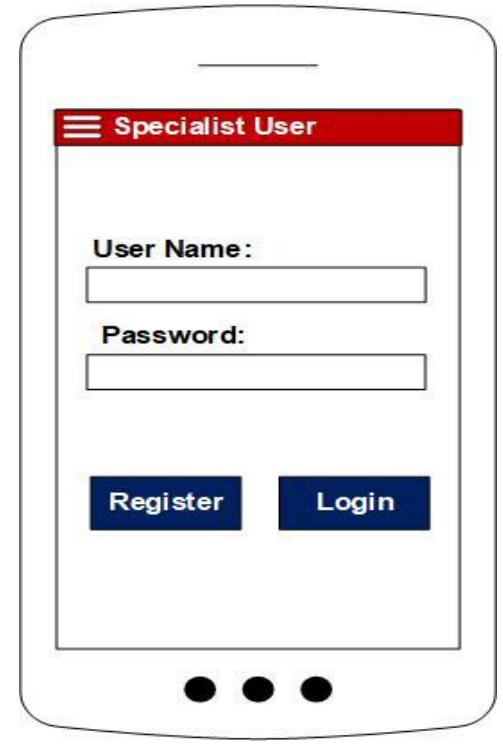

Figure 6. Login interface for specialist



Figure 7. Specialist interface profile

\subsubsection{Health care mobile platforms environments}

Usually in the mobile developments, the developers using a specific programming language and specific platform for a target mobile operating system and then will do the same if the client request the application to work on different mobile system operating systems, clearly the application will link to one singular environment alone as shown in Figure 8, in this study we proposed to use cross platform mobile development for mobile application instead of using mobile native development as it is will be very useful tool to increase the speed of development and decrease the costs and time for development $[14,15]$.

Cross platform development refers to a single code can able to deploy it on different operating systems. E.g. IOS, Android, windows, it could be develop one code in one platform and able to deploy it for multiple mobile operating systems, as shown in Figure 8. In different way the native development the code will link to one singular platform and one mobile operating system and this will cause wasting cost, time and efforts to do single code development for every single mobile operating system as shown in Figure 9. Cross platform it has become very necessary for any mobile application and then will able to send the applications to different various networks and use it on multiple different operating systems [3].

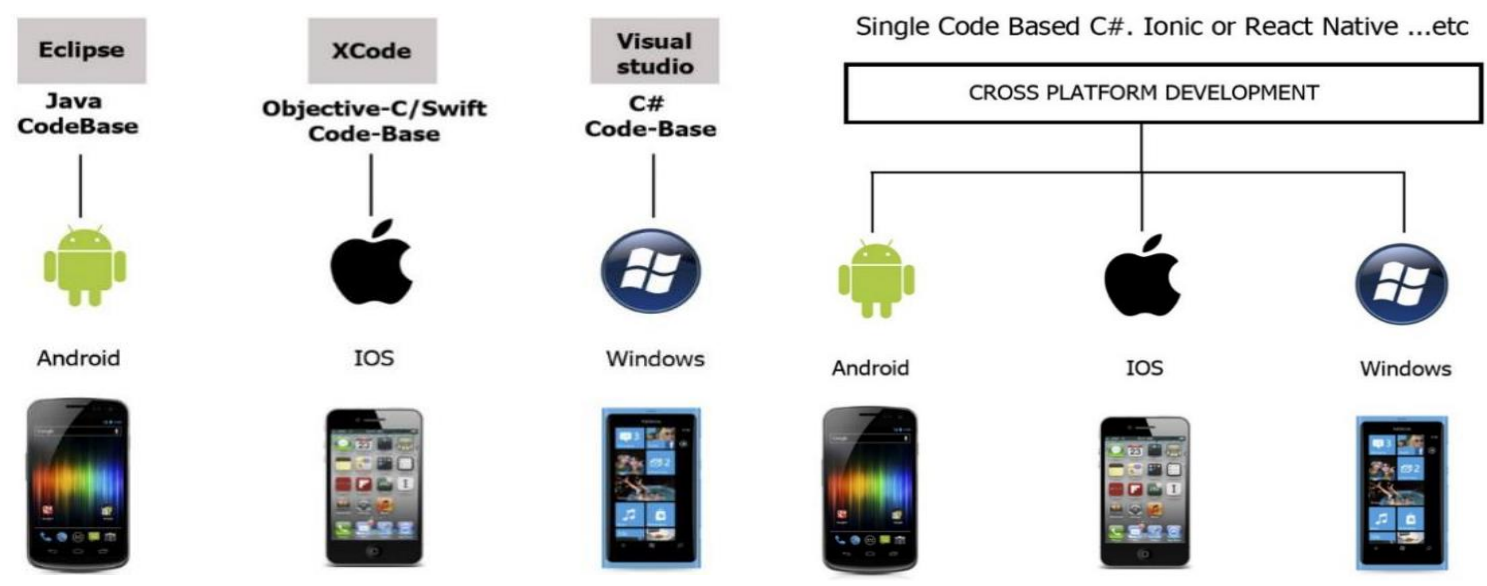

Figure 8. Native platforms developments

Figure 9. Cross Platform development 


\section{RESULTS AND ANALYSIS}

The MCCEH model consists:

a. Database contains all required data tables (emergencies canters, specialists, nurses...etc.) hosted in a cloud server.

b. Develop website to manage the model system

1) Insert/edit/delete emergency center details.

2) Insert/edit/delete nurses.

3) Send notification or message to any determined specialist.

c. Develop mobile application to emergency cases.

The MCCEH model involve to most important medical systems as it starts with login interface to know the user is specialist or normal user, if the user is a normal user then redirect to search interface and other services but if is a specialist then will redirect the user to login/registration with his own account.

\section{CONCLUSION}

Day by day utilization of cloud computing technologies is increasing in every part of the world; very few studies have focused on its impact on. This paper presented a mobile cloud computing for emergency health care (MCCEH) model. In this paper, reduce the responding time of emergency health care to safe the patient life and proposed to use cloud computing server because the cloud computing is reliably flexible, scalable to ensure that health care services won't be interrupted for any reasons and give high performance that allow to user to get the search result within a minute of the time, The process will start from mobile application with searching for nearest medical center or specialist; will get the data from cloud server. In addition, the user could be able to sort the results by availability time, specialization category or previous feedback and voting rates it could help the user to choose the better experience specialist, MCCEH model architecture consists database hosted in cloud server, mobile and web application. The paper focused to manage healthcare model and reduce responding time using cloud computing and cross platform. This paper can be developed for future work to implementing video streaming call, it may help to get Initial diagnosis and give some tips may help the patient.

\section{ACKNOWLEDGMENT}

This work is partially funded by International Islamic University Malaysia (IIUM) Publication RIGS grant no. P-RIGS19-003-0003.

\section{REFERENCES}

[1] A. Nirabi and S. A. Hameed, "Mobile Cloud Computing For Emergency Healthcare Model:Framework," 2018 7th International Conference on Computer and Communication Engineering (ICCCE), Kuala Lumpur, 2018, pp. 375 379.

[2] Chyu MC, Austin T, Calisir F, Chanjaplammootil S, Davis MJ, Favela J, Gan H, Gefen A, Haddas R, HahnGoldberg S, Hornero R. "Healthcare engineering defined: a white paper". Journal of healthcare engineering. 2015;6(4):635-48.

[3] Boyd A, Chambers N, French S, Shaw D, King R, Whitehead A. "Emergency planning and management in health care: priority research topics". Health Systems. 2014 Jun 1; 3(2):83-92.

[4] Ebin J, "Patient safety and quality in healthcare", Sagepub journal, January 29, 2014.

[5] M. Latif, Y. Lakhrissi, E. H. Nfaoui and N. Es-Sbai, "Cross platform approach for mobile application development: A survey," 2016 International Conference on Information Technology for Organizations Development (IT4OD), Fez, 2016, pp. 1-5.

[6] H. Alshareef and D. Grigoras, "First responder help facilitated by the mobile cloud," 2015 International Conference on Cloud Technologies and Applications (CloudTech), Marrakech, 2015, pp. 1-8.

[7] Hameed SA, Bt Mohd Sharifudeen SS, Nuh NH, Bt Salim NH, Hassan A, Khalifa O. "Web-based database and SMS to facilitate healthcare medical emergency". In Proceedings of the Fourth Australasian Workshop on Health Informatics and Knowledge Management Australian Computer Society, Inc. Volume 1202011 Jan 17 (pp. 37-46).

[8] Shah CH, Ismail IM, Mohsin SS. "Ambulance response time and emergency medical dispatcher program: a study in Kelantan, Malaysia". Southeast Asian J Trop Med Public Health. 2008 Nov 1; 39(6):1150-4..

[9] Rajesh Kumar D and ManjupPriya S, "Cloud based M-Healthcare emergency using SPOC," 2013 Fifth International Conference on Advanced Computing (ICoAC), Chennai, 2013, pp. 286-292.

[10] Raj PH, Jelciana P,Kumar PR,. "Exploring data security issues and solutions in cloud computing". Procedia Computer Science. 2018 Jan 1; 125: 691-7.

[11] Almorsy, M., Grundy, J., \& Müller, I. "An analysis of the cloud computing security problem". arXiv preprint arXiv:1609.01107. 2016. 
[12] Hanen J, Kechaou Z, Ayed MB. "An enhanced healthcare system in mobile cloud computing environment". Vietnam Journal of Computer Science. 2016 Nov 1; 3(4):267-77.

[13] Dinh HT, Lee C, Niyato D, Wang P. "A survey of mobile cloud computing: architecture, applications, and approaches". Wireless communications and mobile computing. 2013 Dec 25; 13(18):1587-611.

[14] Gunawan TS, Mutholib A, Kartiwi M. "Design of Automatic Number Plate Recognition on Android Smartphone Platform”. Indonesian Journal of Electrical Engineering and Computer Science. 2017 Jan; 5(1): 99-108.

[15] Griffith C. Mobile App Development with Ionic, Revised Edition: Cross-Platform Apps with Ionic, Angular, and Cordova. " O'Reilly Media, Inc."; 2017 Aug 18.

\section{BIOGRAPHIES OF AUTHORS}

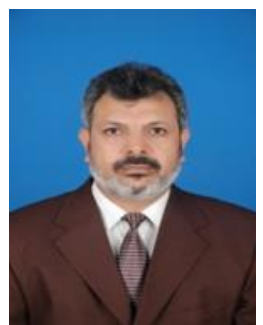

Dr. Shihab A. Hameed is a Full Professor of Computer and Information Engineering in Department of Electrical and Computer Engineering, IIUM University Malaysia. He is a Senior Member in several professional Societies (IEEE, IACSIT, IACSE, ARISE). He is a Member of Board of Study for Computer and Information Engineering Program. Prof Hameed obtained his Ph.D in Computer, Software Engineering from UKM University. He has More than Thirty Five years of Industrial and Educational Experience. His Research Interest is Mainly in Software Engineering and Quality, Healthcare and Medical Applications, Multimedia and Mobile Apps, Professional Ethics, Green ICT and E-Waste, Surveillance and Monitoring Systems. Prof Hameed Supervising Tens of Ph.D and Master Students, Leading or participating in Funded Research Projects and Research Groups, He has more than 200 Publications including Books, Chapters of Books, Research Papers Published in Indexed or Referred Journals and International Conferences. He is participating in organizing and chairing Committees for Tens of International Conferences. Prof Hameed Granted a Patent and obtains more than Twenty Five Medals and Rewards for Innovative and advanced Research work.

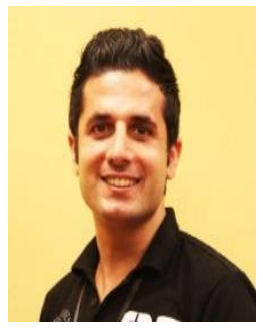

Ali Nirabi is a Post Graduate student pursuing her Master of Science in Computer and Information Engineering, at the department of electrical and Computer Engineering, International Islamic University Malaysia.

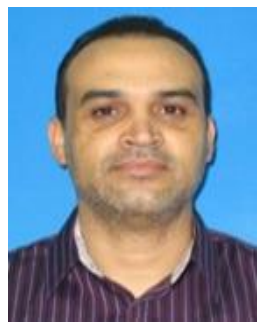

Mohamed H. Habaebi (M'99-SM'16) received his first degree from the Civil Aviation and Meteorology High Institute (CAHI), Libya ('91), his MSc in Electrical Engineering from Universiti Teknologi Malaysia ('94), and his Ph.D. in Computer and Communication System Engineering from Universiti Putra Malaysia ('01). He is currently an Associate Professor and the Post Graduate Academic Advisor at the Department of Electrical and Computer Engineering, International Islamic University Malaysia. He heads the research works on Internet of Things at the department. He has supervised many Ph.D. and M.Sc. students, published more 120 articles and papers and sits on editorial board of many international journals. He is actively publishing in M2M communication protocols, wireless sensor and actuator networks, cognitive radio, small antenna system \& radio propagation and wireless communications \& network performance evaluation. He is an active member of IEEE and an active reviewer to many international journals.

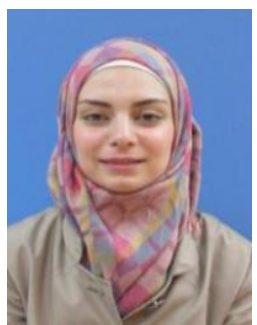

Alaa Haddad is a PG student pursuing her Master of Science in Computer and Information Engineering, at the department of electrical and Computer Engineering, International Islamic University Malaysia. 